\title{
The method of adaptive selection of a wireless access network in a heterogeneous environment based on the theory of fuzzy sets
}

\author{
Andriy Masiuk ${ }^{1, \mathrm{a}}$, Mykhailo Klymash ${ }^{1, \mathrm{~b}}$, Mykola Beshley ${ }^{1,{ }^{*} \text {, }}$ Ivan Demydov ${ }^{1, \mathrm{~d}}$ and Oleksiy \\ Panchenko ${ }^{1, \mathrm{e}}$ \\ ${ }^{1}$ Department of Telecommunications, Lviv Polytechnic National University, Lviv, Ukraine \\ a.masyukand@gmail.com,b.mklimash@lpnu.ua,c.beshlebmi@gmail.com, \\ d.ivan.demydov@gmail.com,e.oleksij@gmail.com \\ *corresponding author
}

Keywords: Vertical handoff, Heterogeneous wireless networks, Multi-criteria, QoS fuzzy logic.

Abstract: The method of choosing a wireless access network in a heterogeneous environment based on the theory of fuzzy sets has been improved. This method allows for a centralized, well-founded decision to carry out a horizontal-vertical handover procedure based on a group of QoS-dependent criteria that depend directly on the properties of the radio interface of the network system. The method also allows adaptation decision-making rules, depending on different telecommunication conditions and network policies. A simulation model of the heterogeneous network has been developed. The model allows to automate investigation of the proposed method of selecting an access node based on the theory of fuzzy sets. The model enables configuration of a large number of simulation parameters using auxiliary mathematical models, in particular for describing and predicting user traffic processes.

Original article, Published date: 2018-05-22

DOI: 10.23977/iotea.2017.31002

ISSN: ISSN 2371-8609 (Print), ISSN 2371-8617 (Online)

https://www.clausiuspress.com/journal/IoTEA.html

\section{Introduction}

In a context of rapid global growth in traffic, the next generation mobile network should focus on customer needs to improve service quality. In order to adapt to the growing flow of information, 
modern approaches such as expanding the spectrum and deploying more base stations in the mobile network will no longer be relevant in terms of cost, scalability and flexibility. Such an increase in information traffic, due in large part to mobile video, social media, and various Internet of things services, forces mobile operators to seek innovative ways to manage networks in a context of limited spectral and backbone resources[8, 15] Telecommunication companies face the challenge of building networks that would be integrated at the most diverse levels, combining different standards and technologies, providing a seamless transition from one standard to another, as well as from one technology to another. These networks are called heterogeneous [9-12]. However, in conditions of heterogeneity and co-operation of many operators, the priority task is to effectively manage common radio resources and ensure the transparent movement of the subscriber, including such mechanisms as mobility support, handover, QoS, security and charging. Handover in a heterogeneous network is a key feature that allows a subscriber to move seamlessly online. In this case, the horizontal handover is a sufficiently researched procedure and is usually based on the received signal strength (RSS). While the vertical handover is a more complex procedure due to the heterogeneous nature of different mobile networks, it needs to be improved to efficiently utilize network resources, maximize the quality of the services provided and user satisfaction [1-5, 17]. However, in most of the known works, the criteria for choosing radio access networks are limited to a few parameters that do not allow for many indices and parameters to be taken into account during the evaluation process and dynamically adapted to decision-making rules. To do this, is necessary to consider transitioning from the current architecture of mobile networks to a new paradigm, based on gathering and storing information in the datacenters for its further analysis and decision-making. That is why the topical issue is the management of networks managed by large data in a cloud environment, since data growth is still a major challenge for today's mobile infrastructure. However, for heterogeneous future networks, it should be noted that the deployment of distributed cloud infrastructure around each base station can lead to an increase in cost compared to a centralized solution, considering the presence of hundreds of cells in the mobile operator's network. Thus, the expansion of the spectrum of services, infrastructure and increasing volumes of traffic leads to solving the scientific task of increasing the efficiency of the wireless heterogeneous network and improving the quality of service for users by improving the method of adaptive selection of radio access network.

\section{Methods and Techniques}

The proposed method of adaptive selection of an access network in a heterogeneous environment includes three main components (fig. 1): data collection, data normalization and decision making on switching [6-7]. 


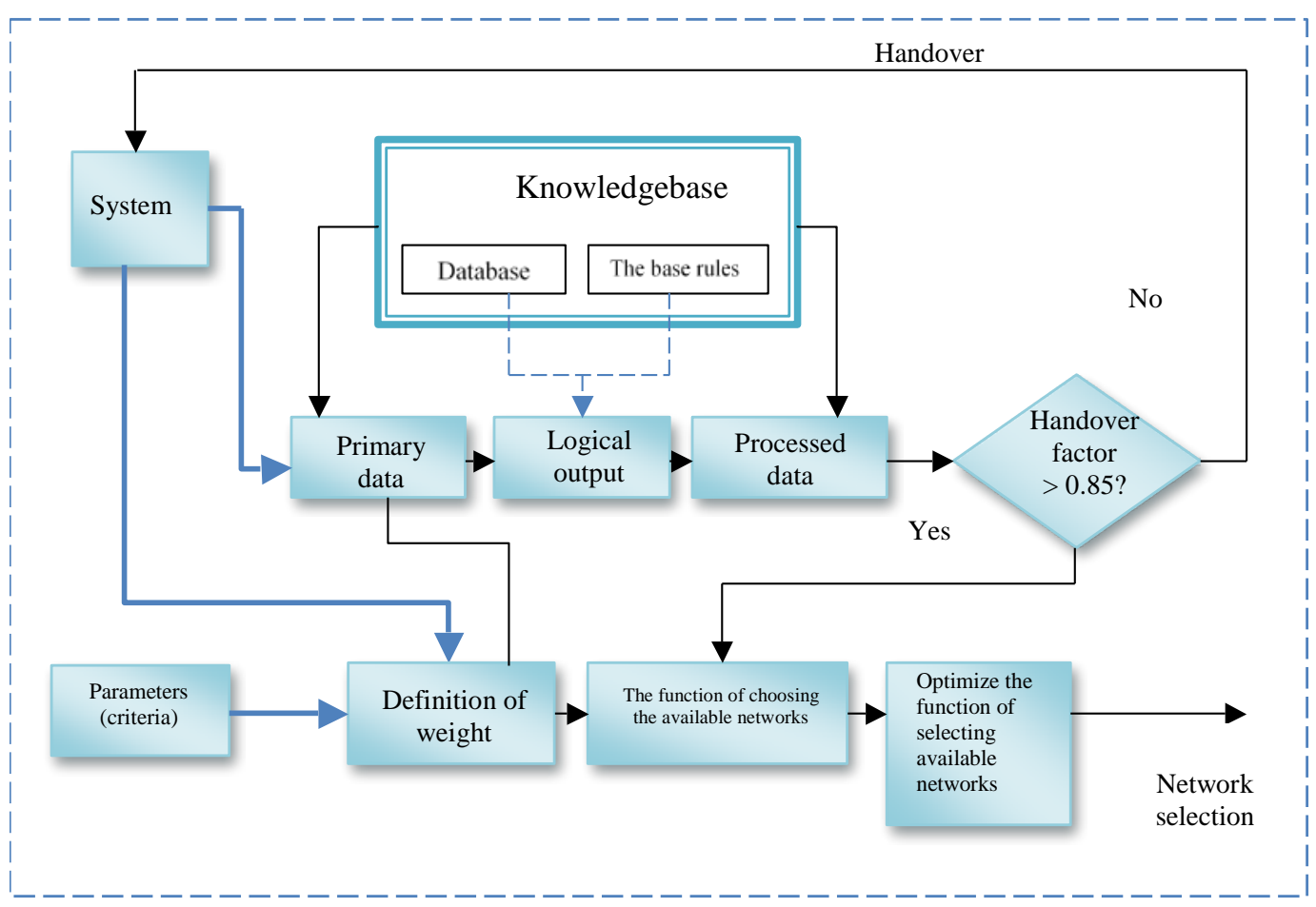

Figure 1: Block scheme of the method of adaptive selection of a wireless access network

The task of this study includes not only balancing the load between different types of BSs, but also improving the quality of customer service by Internet service providers and cellular operators.

To perform intelligent service transmission decisions in a heterogeneous environment and provide a non-disturbing vertical transfer of service, the following parameters that are presented in table. 1 should be considered:

Table 1. Parameters evaluation for handover initiation process with Likert scale

\begin{tabular}{|c|c|c|c|c|c|c|}
\hline SCALE & $\mathbf{1}$ & $\mathbf{2}$ & $\mathbf{3}$ & $\mathbf{4}$ & $\mathbf{5}$ & $\begin{array}{c}\text { The Min/Max } \\
\text { Quantities }\end{array}$ \\
\hline Load, [\%] & $70-100$ & $50-70$ & $40-50$ & $30-40$ & $0-30$ & $0-100$ \\
\hline Cost & $1-2$ & $2-3$ & $3-4$ & $4-8$ & $8-10$ & $1-10$ \\
\hline Security & $1-2$ & $2-4$ & $4-6$ & $6-8$ & $8-10$ & $1-10$ \\
\hline Ms-Velocity, [km/h] & $<120$ & $80-120$ & $60-80$ & $40-60$ & $0-40$ & $0-160$ \\
\hline Delay,[ms] & $<300$ & $200-300$ & $100-200$ & $50-100$ & $10-50$ & $10-500$ \\
\hline Jitter, [ms] & $<30$ & $20-30$ & $10-20$ & $5-10$ & $1-5$ & $10-30$ \\
\hline Packet Loss Ratio (PLR), [\%] & $<8$ & $6-8$ & $4-6$ & $3-4$ & $1-3$ & $1-8$ \\
\hline Throughput, [Mbps] & $<0 / 1$ & $0.1-10$ & $1-50$ & $50-100$ & $100-200$ & $0 / 1-200$ \\
\hline RSS, [dbm] & $<-110$ & $-100--$ & $-90--100$ & $-75--90$ & $-55--75$ & $-110--55$ \\
\hline
\end{tabular}

The creation of such an intelligent vertical reception / transmission service algorithm is a rather complicated task due to the complexity of the service delivery and transition chain, the adoption of metrics, the modularity of the service transmission solution, the information inaccuracies, and interpreted issues. In this paper, fuzzy logic is proposed as an effective means for solving these problems, as well as a description of the decision and the logical conclusion were performed. A fuzzy logic applied system in general consists of three parts: normalization of input parameters, 
fuzzy knowledge base and normalized output parameters. As an incomplete method of representing knowledge, fuzzy logic is well suited for solving issues in practice, inaccuracies.

Fuzzy logic is a multi-valued logic that deals with approximate considerations. This adds to the logical (Boolean) or ambiguous logic an important opportunity - to make decisions with imperfect information, that is, information that in one or several ways may be inaccurate, indeterminate, incomplete, inaccurate, or partially valid. Fuzzy logic consists of fuzzy sets and fuzzy set operations are used to draw conclusions on fuzzy logic.

After the normalization procedure, all parameters are translated into linguistic variables (terms) that can be compared directly, since the parameters will be reduced to a single reference database. The decision-making module simply processes the parameters of a wireless network, directly comparing them with the help of a fuzzy knowledge base to derive the optimal solution.

For this method, we first introduce the weight scale of each criterion for the initiator handover in accordance with the Likert scale ( 1 - very inapplicable $(0,0,0.25)$, 2 - inapplicable $(0,0.25,0.75), 3$ medium, $(0.25,0.5,0.75), 4$ - applicable $(0.5,0.75,1)$, 5 - very applicable $(0.75,0.75,1))$. Also, we introduce a five-level scale of linguistic terms to assess the correlation between the relevant indicator of the network system and the criterion (1 - "very low", 2 - "low", 3 - "average", 4 "high", 5 - "very high"), which allows to evaluate the value of the level of the corresponding criterion parameter on the scale of rice.

Lets form empiric coefficients of services importance regarding required quality of service for handover initiation using coefficients $B_{1}, B_{2}, B_{3}$ and $B_{4}$, that can be in a range from 1 to 3 , where higher value means higher importance of specific quality of service parameter for a particular service category.

Table 2. Services significance coefficient relative to others

\begin{tabular}{|c|c|c|c|c|}
\hline S & $\begin{array}{c}\text { PLR } \\
\mathrm{B}_{1}\end{array}$ & $\begin{array}{c}\text { Delay } \\
\mathrm{B}_{2}\end{array}$ & $\begin{array}{c}\text { Jitter } \\
\mathrm{B}_{3}\end{array}$ & $\begin{array}{c}\text { Throu } \\
\text { ghput } \\
\mathrm{B}_{4}\end{array}$ \\
\hline Voice & 2 & 3 & 3 & 1 \\
\hline Videoconference & 2 & 3 & 3 & 2 \\
\hline IPTV & 3 & 2 & 2 & 3 \\
\hline Internet data & 3 & 1 & 1 & 1 \\
\hline Interactive data & 2 & 2 & 1 & 1 \\
\hline VoD & 2 & 2 & 2 & 3 \\
\hline VoIP & 3 & 2 & 1 & 1 \\
\hline
\end{tabular}

First, an influence of increasing load of each cell in all access network technologies on parameters that affect selection of access network when doing intellectual vertical handover is determined. Process of selection of optimal network will be performed in monitoring system of heterogeneous network using cloud technology and fuzzy logic

Table 3. Coefficient of parameter importance

\begin{tabular}{|c|c|c|c|}
\hline $\mathbf{P}$ & $\begin{array}{c}\text { GSM } \\
\mathbf{B}_{\mathbf{1}}\end{array}$ & $\begin{array}{c}\text { LTE } \\
\mathbf{B}_{\mathbf{2}}\end{array}$ & $\begin{array}{c}\text { UMTS } \\
\mathbf{B}_{\mathbf{3}}\end{array}$ \\
\hline Cost & 1 & 2 & 2 \\
\hline Security & 1 & 2 & 1 \\
\hline RSS & 2 & 2 & 2 \\
\hline Load & 2 & 1 & 2 \\
\hline Ms-Velocity & 1 & 2 & 1 \\
\hline
\end{tabular}


Let's introduce parameter of node utilization capacity for particular wireless technology where $\mathrm{m}$ is a number of node of access system located in space, and $\mathrm{n}$ - technologies of wireless access. Let's assume that $n=1$ for LTE, $n=2$ for GSM, $n=3$ for UMTS.

Table 4. Services significance coefficient relative to others

\begin{tabular}{|l|l|l|}
\hline & \multicolumn{1}{|c|}{ Load } & \\
\hline Jitter & Increasing $\rho \uparrow$ & Decreasing $\mathrm{j} \downarrow$ \\
\hline Cost & Increasing $\rho \uparrow$ & Increasing $\mathrm{c} \uparrow$ \\
\hline Security & Increasing $\rho \uparrow$ & Const $\mathrm{s} \leftrightarrow$ \\
\hline Ms-Velocity,[km/h] & Increasing $\rho \uparrow$ & Decreasing $\mathrm{v} \uparrow$ \\
\hline Delay,[ms] & Increasing $\rho \uparrow$ & Increasing $\mathrm{d} \uparrow$ \\
\hline Packet Loss Ratio [\%] & Increasing $\rho \uparrow$ & Increasing $\mathrm{p} \uparrow$ \\
\hline Throughput,[Mbps] & Increasing $\rho \uparrow$ & Decreasing $\mathrm{t} \downarrow$ \\
\hline RSS, [dbm] & Increasing $\rho \uparrow$ & Const $\mathrm{r} \leftrightarrow$ \\
\hline
\end{tabular}

After values of wireless node parameters were estimated in the process of node evaluation based on collection of indexes, that characterize QoS by default all weights equal 0,5. In case weight decreasing and $\rho_{m n}$ increasing, weight of parameter is calculated in accordance to table 3 by formula:

$$
w p_{\text {imn }}=0.5+0.5 \cdot \rho_{m n}, i=1 . .4
$$

In case weight decreasing and $\rho_{m n}$ increasing, weight of parameter is calculated in accordance to table 3 by formula:

$$
w p_{\text {imn }}=0.5-0.5 \cdot \rho_{m n}, i=1 . .4
$$

where $i$ is QoS parameter (see table 2) that is taken into account in the process of intellectual vertical handover.

Finally weight is obtained as a result of multiplying parameters weights (1-2) and coefficient of parameter importance regarding to requested service:

$$
w_{i m n}=w p_{i m n} B_{i}, i=1 . .4
$$

Where $B_{i}$ is coefficient of parameters importance regarding to service type requested by user (shown in table 2).

Next normalization of $w_{i m n}$ is performed:

$$
W_{i m n}=w_{i m n} / \sum_{i=1}^{4} w_{i m n},
$$

Respectively evaluation of selected access node $\widetilde{Q}_{m n}$ is calculated regarding to selected technology in accordance with parameters evaluations obtained in table 1 , that immediately affect QoS:

$$
\begin{aligned}
& \tilde{Q}_{m n}=\left(q_{1}, q_{2}, q_{3}\right)_{m n}=\sum_{i=1}^{4}\left(W_{i m n} \times \tilde{L}_{i m n}\right) \\
& q_{j_{m n}}=\sum_{i=1}^{4}\left(W_{i_{m n}} \times l_{i j_{m n}}\right), \quad(j=1,2,3 ; m=1,2,3 ; n=1,2),
\end{aligned}
$$


where $q_{1}, q_{2}, q_{3}$ are bottom level of general evaluation $\tilde{Q}$, its basic value and top level respectively, $\tilde{L}_{i m n}=\left(l_{i 1}, l_{i 2}, l_{i 3}\right)_{m n}$ - three-cornered fuzzy number that characterizes indicator of node parameter by $i$ criteria for node of access system $m$ of technology $n$. Here $l_{i 1}, l_{i 2}, l_{i 3}$ - bottom level of linguistic variable, its basic value and top level respectively to TFN format (Triangular Fuzzy Number) see (Fig. 5).

After determination of values of access node parameters in the process of node evaluation based on collection of indexes that characterize physical link to make an appropriate decision on handover procedure by air interface circumstances we operating with following parameters for which by default all weights equal to those defined in table 4: RSS, Load, Security, Velocity, Cost.

In case of increasing weight and increasing of $\rho$, the weight of parameter is determined in accordance with table 3 by formula:

$$
p l p_{k m n}=0.5+0.5 \cdot \rho_{m n}
$$

In case of decreasing weight and increasing of $\rho$, the weight of parameter is determined in accordance with table 3 by formula:

$$
p l p_{k m n}=0.5-0.5 \cdot \rho_{m n},
$$

where $\mathrm{k}$ is a parameter of physical radio interface that is taken into consideration in the process of vertical handover.

Resulting weight is obtained as a result of multiplying parameters evaluations based on table 1 and coefficient of parameter importance according to table 4 for each technology:

$$
p l_{k m n}=p l p_{k m n} B_{k}, k=1 . .5 \text {, }
$$

where $k$ is a parameter that is taken into consideration in the process of vertical handover.

where $B_{k}$ is an importance coefficient of parameters respectively to wireless access technology type (shown in table 4).

Next normalization of $w_{k m n}$ is performed:

$$
P L_{k m n}=p l_{k m n} / \sum_{i=1}^{5} p l_{k m n},
$$

Respectively the selected node evaluation $\widetilde{P}_{m n}$ is calculated regarding to selected technology according to parameters evaluation that are obtained from table 1 that immediately affect quality of radio interface:

$$
\begin{gathered}
\tilde{P}_{m n}=\left(p_{1}, p_{2}, p_{3}\right)_{m n}=\sum_{i=1}^{5}\left(P L_{k m n} \times \tilde{L}_{k m n}\right) \\
p_{j_{m n}}=\sum_{i=1}^{5}\left(P L_{k_{m n}} \times l_{k j_{m n}}\right), \quad(j=1,2,3 ; m=1,2,3 ; n=1,2),
\end{gathered}
$$

where $p_{1}, p_{2}, p_{3}$ are bottom level of general evaluation $\widetilde{P}$, its basic value and top level respectively, $\tilde{L}_{k m n}=\left(l_{k 1}, l_{k 2}, l_{k 3}\right)_{m n}$ is triangular fuzzy number TFN that characterizes indicator of node parameter by $k$ criteria for the node of $m$ access system based of $n$ technology. Here $l_{k 1}, l_{k 2}, l_{k 3}$ is bottom level of linguistic variable, its basic value and top level respectively to TFN format (Fig.5).

This way generalized evaluation of access node is determined as: 


$$
\begin{aligned}
& \tilde{R}_{m n}=\left(r_{1 m n}, r_{2 m n}, r_{3 m n}\right)=1 / 2 \times\left(\tilde{Q}_{m n}+\tilde{P}_{m n}\right) \\
& =1 / 2 \times\left(q_{1 m n}+p_{1 m n}, q_{2 m n}+p_{2 m n}, q_{3 m n}+p_{3 m n}\right)
\end{aligned}
$$

Lets conduct defuzzification of obtained fuzzy number in accordance with [6]:

$$
R_{m n}=1 / 3 \times \sum_{t=1}^{3} r_{t m n}
$$

\section{Results and Discussion}

Using the software implementation of the simulation model, we can implement not only all the necessary simulation functions, but also provide proper control over its process. The software allows us to use a graphical user interface, dynamically modify the model parameters, and thus evaluate the system behavior in a particular situation that may occur in a real service network platform. In addition, the software through the graphical interface allows you to watch in real time all the parameters of the model that can be executed using graphs, charts, lists and tables.

To study the operation of a mobile network system with a high level of user mobility, it is necessary to develop an imitation model using a large number of parameters and characteristics that allow describing existing network technologies using, respectively, mathematical, predictive and optimization models. Thus, in this paper a model of a heterogeneous network platform of wireless access, which automates the proposed method, was developed.

The simulation model contains a block of user behavior simulation that includes traffic generation. Formed objects-users are transferred to the "user manager". If the user only registers in the network and does not have active sessions, he is noted in the graphical interface of the model, as a thick gray dot. If the user has an active connection to the network, then it is marked with a color dot with the color depending on which network system it is connected to: GSM - green, LTE - blue, UMTS - pink color. The user in this model is presented as an object with a set of parameters such as: activity duration, motion vector, speed, location, distance to base stations (network access nodes), signal level from each base station (LTE, UMTS and GSM) (RSS) and other additional parameters necessary for the operation of the model.

To develop such software in this work, the programming language $\mathrm{C}++$ is based on Borland Builder $\mathrm{C}++6.0$ development environment was used. The main window of the program that implements the simulation in accordance with the created simulation model is shown in Fig. 6. The interface contains a component for displaying the use of three base stations of GSM, UMTS type and three base stations of LTE type. Time characteristics of GSM,UMTS and LTE performance under the conditions of overload in heterogeneous system depicted in fig.3-5. 


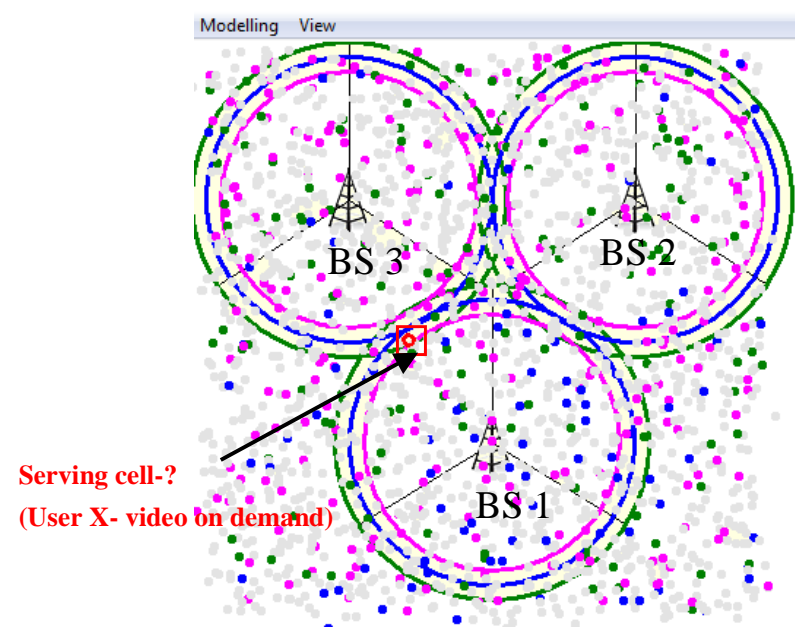

Figure 2: Users mobility modeling according to Brownian law

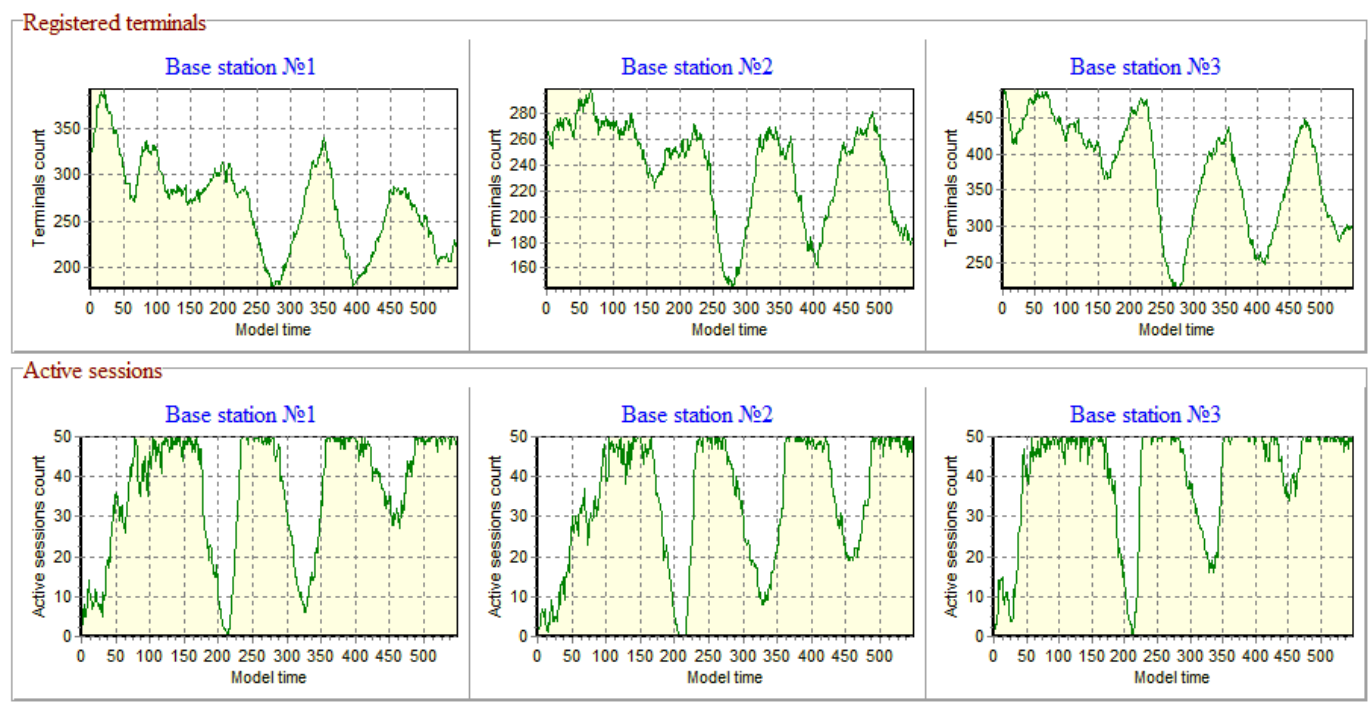

System characteristics

Base station №2: number of redirected connections: 271

Base station №3: number of redirected connections: 932

Total count of successful connections: 3444 , lost $=0$

Total count of redirected connections: 1599

Figure 3: Time characteristics of GSM performance under the conditions of overload in heterogeneous system 

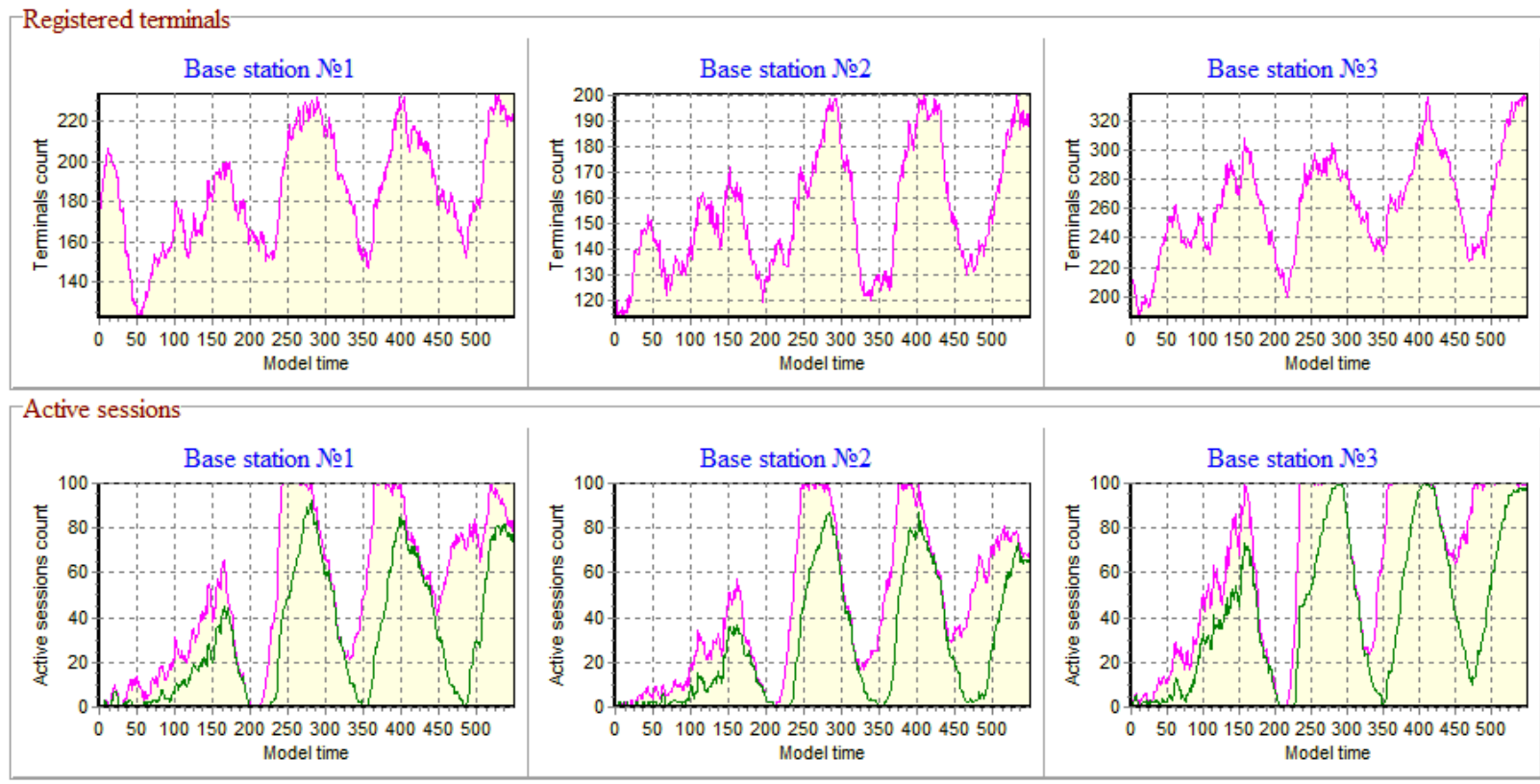

Figure 4: Time characteristics of UMTS performance under the conditions of overload of GSM system in heterogeneous system
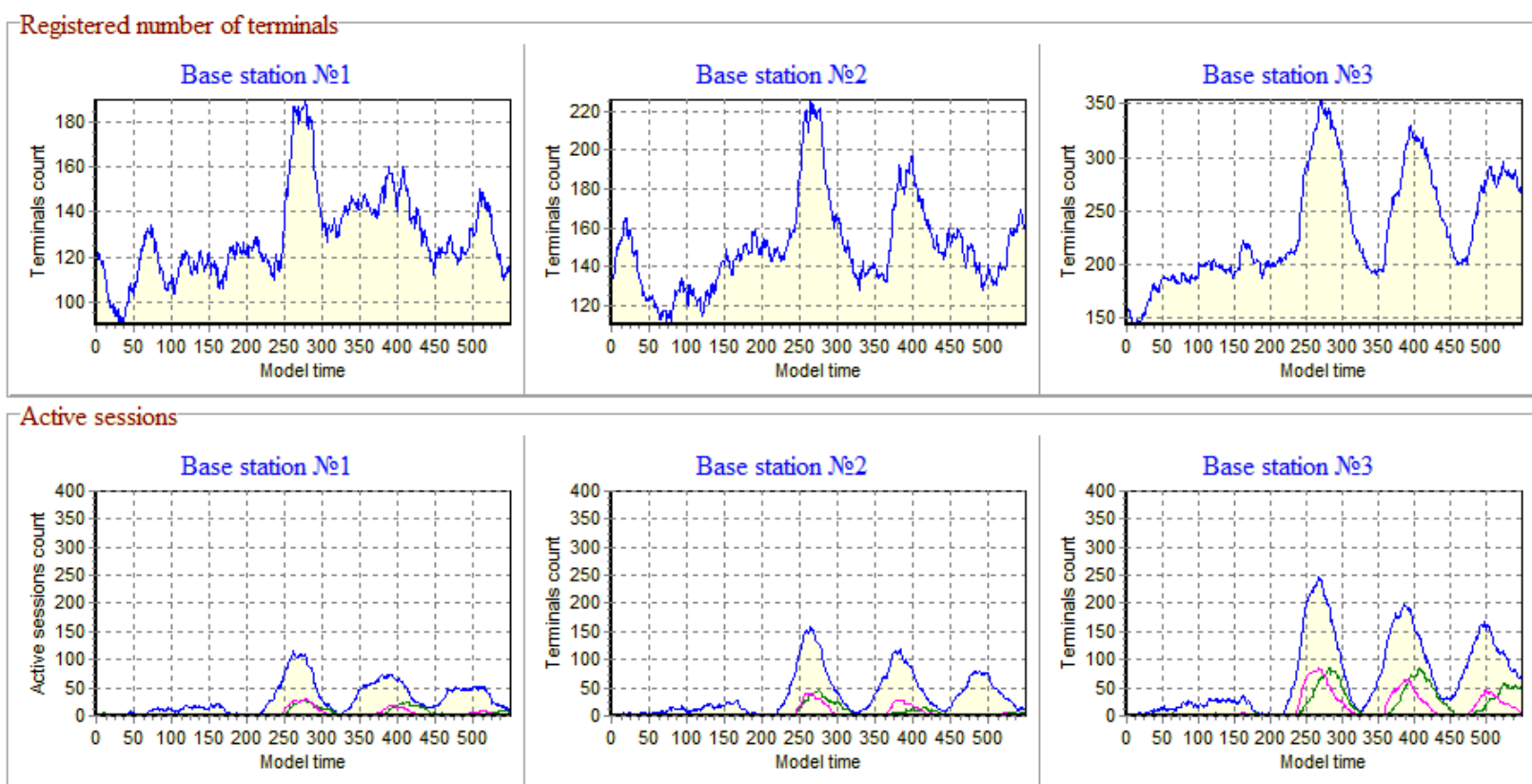

Figure 5: Time characteristics of LTE performance under the conditions of overload of UMTS and GSM in heterogeneous system

Fig. 6 demonstrate the utilization of cloud server that hold the software that is responsible for conducting intellectual vertical handover. Thus, using the capabilities and means of cloud service platforms, such calculations of the optimal choice of network system is provided within a few milliseconds. 


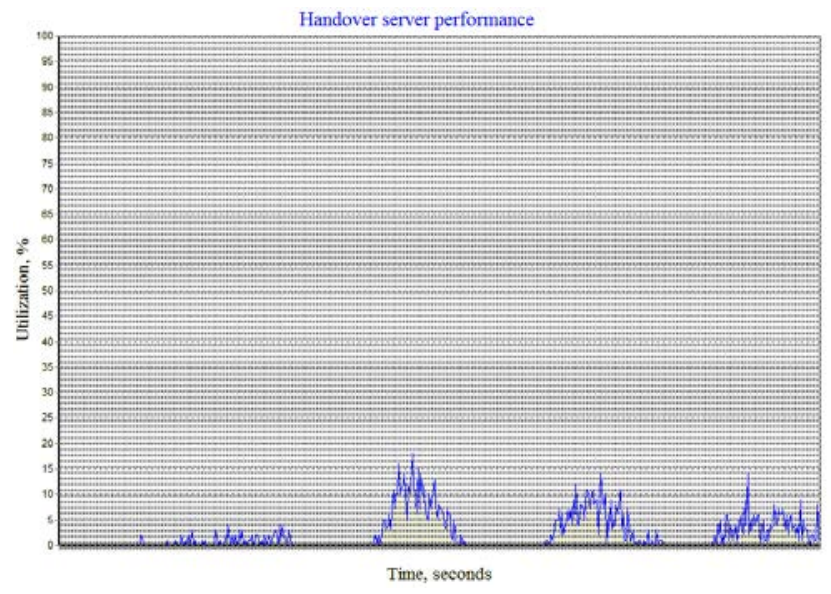

Figure 6: Utilization of cloud server that hold the software that is responsible for conducting intellectual vertical handover

For each base station there is a program interface graph that displays registered and active users. In order to compare the performance of the system without an intelligent vertical handover and with it, the program model has the ability to disable this algorithm. Thus, the theory of fuzzy sets quantitatively demonstrates the difference between the QoS (delay and jitter) of real-time video services with a homogeneous and heterogeneous network [16]. For standard handover (serving cell is UMTS BS1) the delay is 1,9 sec.(fig.7a) and jitter is 1,4 sec. (fig.7b). For proposed handover (serving cell is LTE BS1) the delay is 115 ms. (fig.7c) and jitter is 20 ms. (fig.7d).

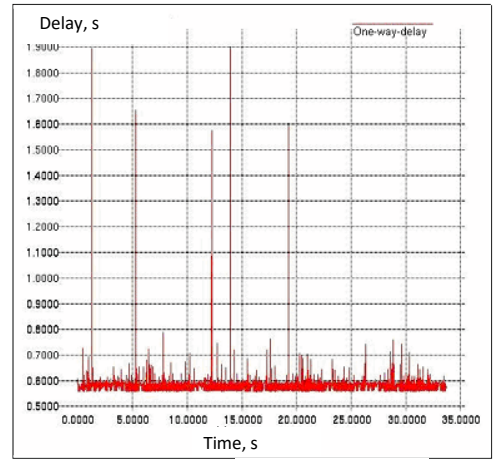

a)

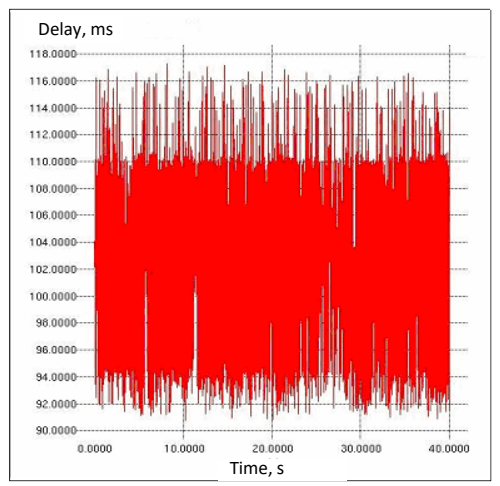

c)

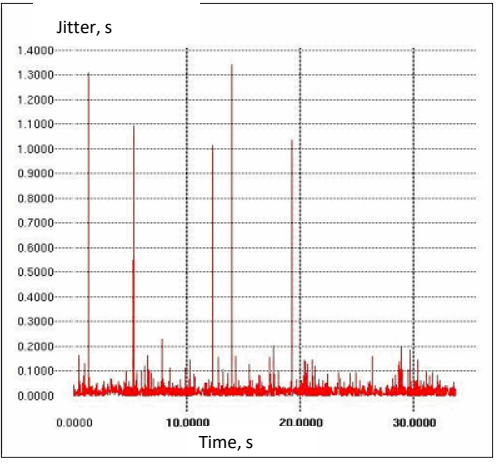

b)

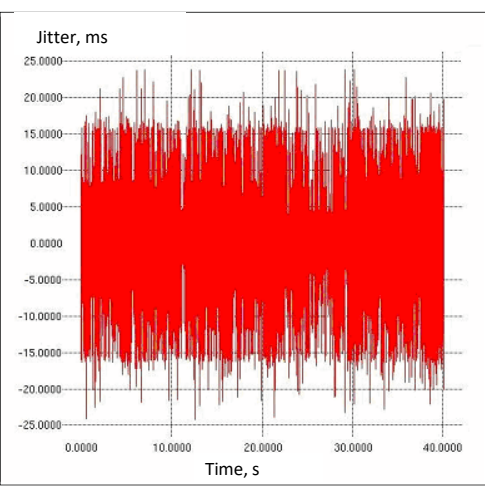

d)

Figure 7: Video delay (a), jitter (b) for standard handover and video delay (c), jitter(d) for proposed handover 


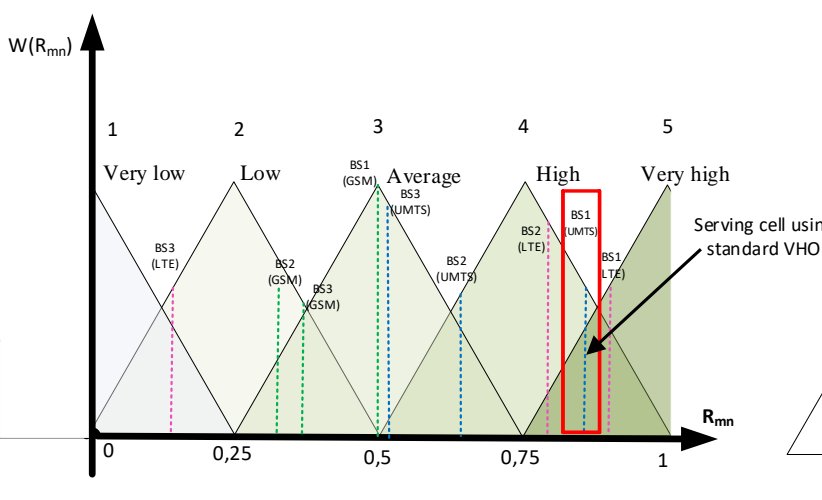

a)

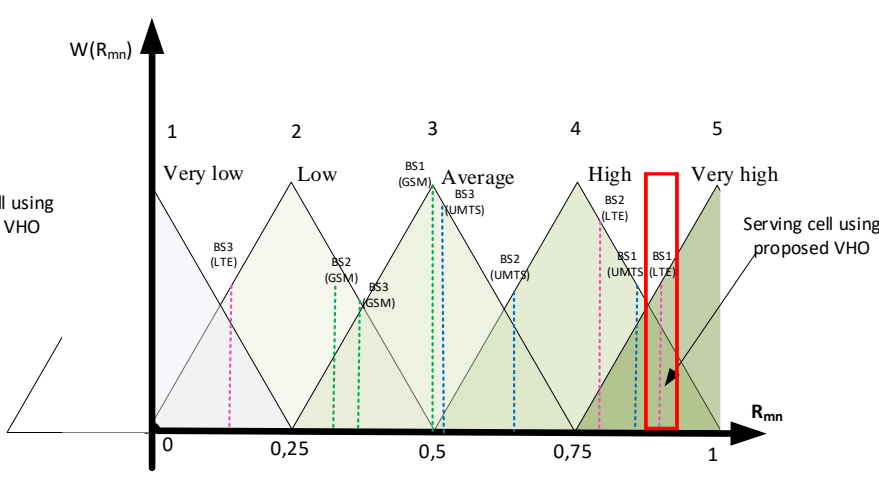

b)

Figure 8: Optimal cell determination by using Likert scale for standard handover a) and proposed handover b)

Based on the proposed solutions the following results was obtained for the access node BS1 (LTE network system fig.8b). The proposed model of the heterogeneous network allows to investigate the process of optimization of the network infrastructure of a mobile operator by redistributing network resources and balancing the load by implementing the algorithm of the vertical handover.

\section{Conclusions}

To solve the problem of finding the optimal solution for a resource redistribution procedure when servicing users of wireless platforms of wireless access, a centralized method of choosing an access network for managing the handover has been proposed. The proposed method avoids ambiguity in the interpretation of fuzzy, ambiguous and subjective judgments in the process of multicriteria optimization. To study the processes of functioning of real heterogeneous network systems under the conditions of high mobility of users, an simulation model has been developed. This model includes the proposed mathematical model of the decision-making process for a vertical handover based on the use of fuzzy logic methods. This allows to configure a large number of simulation parameters using auxiliary mathematical models, in particular for describing and predicting user traffic (mobility), as well as distributing radio waves to the terminal equipment. The choice of the optimal network access node based on the solution of the multi-criteria decisionmaking task for the handover is non-trivial, since the results of this solution are influenced simultaneously by several dynamic and variables and interconnected factors. Therefore, their aggregation in accordance with the approaches proposed in the work is required. In an ambivalent situation, when the user is on the boundary of the cell's coverage, the proposed algorithm allowed to make a reasonable decision on the procedure of vertical handover, based on a group of QoSdependent criteria.

\section{Acknowledgements}

This research is supported by the project entitled "Methods for designing the heterogeneous information and communication system to deploy the software defined multi-purpose 5G networks", funded by Ukrainian government. 


\section{References}

[1] J. He, J. Rexford, and M. Chiang, "Design principles of manageable networks", Princeton University Computer Science, Technical Report TR-770-06, Oct. 2006.

[2] M. Lahby and A. Sekkaki, "Optimal vertical handover based on TOPSIS algorithm and utility function in heterogeneous wireless networks," 2017 International Symposium on Networks, Computers and Communications (ISNCC), Marrakech, 2017, pp. 1-6.

[3] D. Cheelu, R. B. Madda, C. Y. Chen, P. Venkata Krishna and S. Yenduri, "Intelligent vertical handoff decision strategy based on network performance prediction and consumer surplus value for next generation wireless networks," in IET Networks, vol. 6, no. 4, pp. 69-74, 72017.

[4] S. Sasi, R. D. Daruwala, T. Palav and P. Mule, "A hybrid vertical handoff decision algorithm for seamless mobility in heterogeneous wireless networks," 2016 International Conference on Wireless Communications, Signal Processing and Networking (WiSPNET), Chennai, 2016, pp. 1921-1923.

[5] J. Chen, Z. Wei, Y. Wang, L. Sang and D. Yang, "A service-adaptive multi-criteria vertical handoff algorithm in heterogeneous wireless networks," 2012 IEEE 23rd International Symposium on Personal, Indoor and Mobile Radio Communications - (PIMRC), Sydney, NSW, 2012, pp. 899-904.

[6] M. Klymash, B. Stryhalyuk, I. Demydov, M. Beshley, M. Seliuchenko. A Novel Approach of Optimum Multi-criteria Vertical Handoff Algorithm for Heterogeneous Wireless Networks // International Journal of Engineering and Innovative Technology (IJEIT) . - 2014. - Volume 4 . - Issue 5(4). - P. 42-52.

[7] Beshley M. SOA quality management subsystem on the basis of load balancing method using fuzzy sets / M. Beshley, M. Klymash, B.Strykhalyuk, O. Shpur, B. Bugil, I. Kagalo // International Journal of Computer Science and Software Engineering (IJCSSE). - 2015 - Volume 4 - Issue 1 - P.10-21

[8] H. Beshley, M. Beshley, T. Maksymyuk and I. Strykhalyuk, "Method of centralized resource allocation in virtualized small cells network with IoT overlay," 2018 14th International Conference on Advanced Trends in Radioelecrtronics, Telecommunications and Computer Engineering (TCSET), Lviv-Slavske, Ukraine, 2018, pp. 1147-1151.

[9] M. Klymash, H. Beshley, M. Seliuchenko and M. Beshley, "Algorithm for clusterization, aggregation and prioritization of M2M devices in heterogeneous 4G/5G network," 2017 4th International Scientific-Practical Conference Problems of Infocommunications. Science and Technology (PIC S\&T), Kharkov, 2017, pp. 182-186.

[10] M. Klymash, H. Beshley, O. Panchenko and M. Beshley, "Method for optimal use of 4G/5G heterogeneous network resourses under M2M/IoT traffic growth conditions," 2017 International Conference on Information and Telecommunication Technologies and Radio Electronics (UkrMiCo), Odessa, 2017, pp. 1-5.

[11] M. Klymash, O. Lavriv, T. Maksymyuk and M. Beshley, "State of the art and further development of information and communication systems," 2016 International Conference Radio Electronics \& Info Communications (UkrMiCo), Kiev, 2016, pp. 1-6.

[12] I. Demydov, M. Seliuchenko, M. Beshley and M. Brych, "Mobility management and vertical handover decision in an always best connected heterogeneous network," The Experience of Designing and Application of CAD Systems in Microelectronics, Lviv, 2015, pp. 103-105.

[13] M. Klymash, M. Beshley and B. Stryhaluk, "System for increasing quality of service of multimedia data in convergent networks," 2014 First International Scientific-Practical Conference Problems of Infocommunications Science and Technology, Kharkov, 2014, pp. 63-66.

[14] A. Masiuk, M. Beshley, O. Lavriv and Y. Deschynskiy, "Common radio resource management model for heterogeneous cellular networks," IEEE International Conference on Modern Problems of Radio Engineering, Telecommunications and Computer Science (TCSET'2016), Feb. 23-26, 2016, Lviv, Ukraine, pp. 661-663.

[15] M. Klymash, M. Beshley, B. Strykhalyuk and T. Maksymyuk, "Research and development the methods of quality of service provision in Mobile Cloud systems," 2014 IEEE International Black Sea Conference on Communications and Networking (BlackSeaCom), Odessa, 2014, pp. 160-164

[16] M. Seliuchenko, M. Beshley, O. Panchenko and M. Klymash, "Development of monitoring system for end-to-end packet delay measurement in software-defined networks," IEEE International Conference on Modern Problems of Radio Engineering, Telecommunications and Computer Science (TCSET'2016), Feb. 23-26, 2016, Lviv, Ukraine, pp. $667-670$.

[17] M. Klymash, R. Savchuk, P. Pozdnyakov and M. Beshley, "The researching and modeling of structures of mobile networks for providing of multiservice radio access," Proceedings of International Conference on Modern Problem of Radio Engineering, Telecommunications and Computer Science, Lviv-Slavske, 2012, pp. 281-282. 